http://jmscr.igmpublication.org/home/ ISSN (e)-2347-176x ISSN (p) 2455-0450

crossref DOI: https://dx.doi.org/10.18535/jmscr/v7i9.130

\author{
Journal Of Medical Science And Clinical Research \\ IGM Publication \\ An Official Publication of IGM Publication
}

\title{
A Rare Case of Dubowitz Syndrome with Polyendocrinopathy
}

\author{
Authors \\ Gaurav Gupta ${ }^{1}$, Dr Ila Pahwa ${ }^{2}$, Dr Hemant Kumar Sharma ${ }^{3}$ \\ ${ }^{1}$ Junior Resident, Department of Medicine, Muzaffarnagar Medical College Muzaffarnagar, Uttar Pradesh, India \\ ${ }^{2}$ Professor \& Head, Department of Medicine, Muzaffarnagar Medical College Muzaffarnagar, Uttar Pradesh India \\ ${ }^{3}$ Professor, Department of medicine, Muzaffarnagar Medical College Muzaffarnagar, Uttar Pradesh India
}

\begin{abstract}
Dubowitz Syndrome - A very rare genetic and developmental disorder, characterized by Microcephaly, Stunted Growth, Small Jaw (Micrognathia), Sparse Hair, Mental Retardation. Symptoms may vary among patients, facial appearance is the key to the diagnosis. H/O Consanguineous Marriage in parents. Thus far only about 200 cases have been reported worldwide since 2002. Here, we report a case of a male, 30 years, presented with Non Healing Ulcer over left foot, with characteristics features of Short Stature, Microcephaly, Microganathia, and High Arched Palate with Polyendocrine involvement like Diabetes Mellitus, Hypothyroidism, and Hypoparathyroidism. H/O Consanguineous Marriage in Parents is present. Prompt diagnosis with regular follow up and supportive care remains the key to the management of this disorder.
\end{abstract}

\section{Introduction}

A very rare genetic and developmental disorder, characterized by microcephaly, stunted growth, small jaw (micrognathia), sparse hair, mental retardation. ${ }^{1}$ Symptoms may vary among patients; facial appearance is the key to the diagnosis. History of consanguineous marriage in parents is present. Thus far only about 200 cases have been reported worldwide since $2002 .^{2}$ The pathogenesis of this syndrome is unknown and is presumed to represent the inherited by autosomal recessive manner. ${ }^{3}$

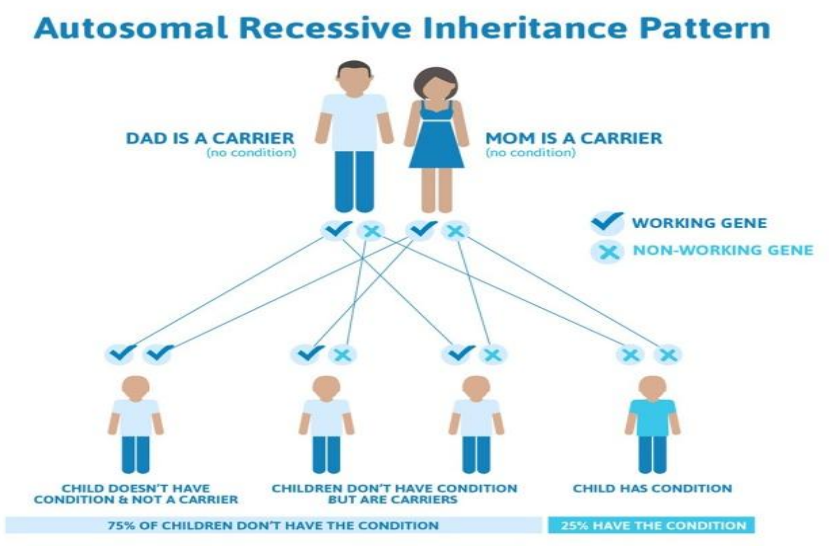

\section{Case History}

Here, we report a case of a male, 30 years born of a consanguineous marriage presented with non healing ulcer over left foot. A general physical examination found with characteristics features of - short stature/ growth failure, small head (microcephaly), small face, small lower jaw 
(micrognathia), high arched palate, prominent ears, hook nose, delayed eruption of teeth with missing teeth, sparse hair, scanty lateral eyebrows, micropthalmia, high pitched voice, genu valgum (knock-knee).

Developmental assessment revealed shy nature with normal intelligence. Ophthalmological and audiological assessment was normal. Endocrinal gland involvement-diabetes mellitus (HbA1c 9.5\%); hypothyroidism (TSH $75.04 \mathrm{Uiu} / \mathrm{ml}, \mathrm{T} 3$ total $0.58 \mathrm{ng} / \mathrm{ml}, \mathrm{T} 4$ total $2-10 \mathrm{ug} / \mathrm{ml}$, anti TPOab positive $100 \mathrm{IU} / \mathrm{ml}$ ); hypoparathyroidism (intact PTH 12.8 pg/ml; calcium 7.6 ; phosphorus 6.64).

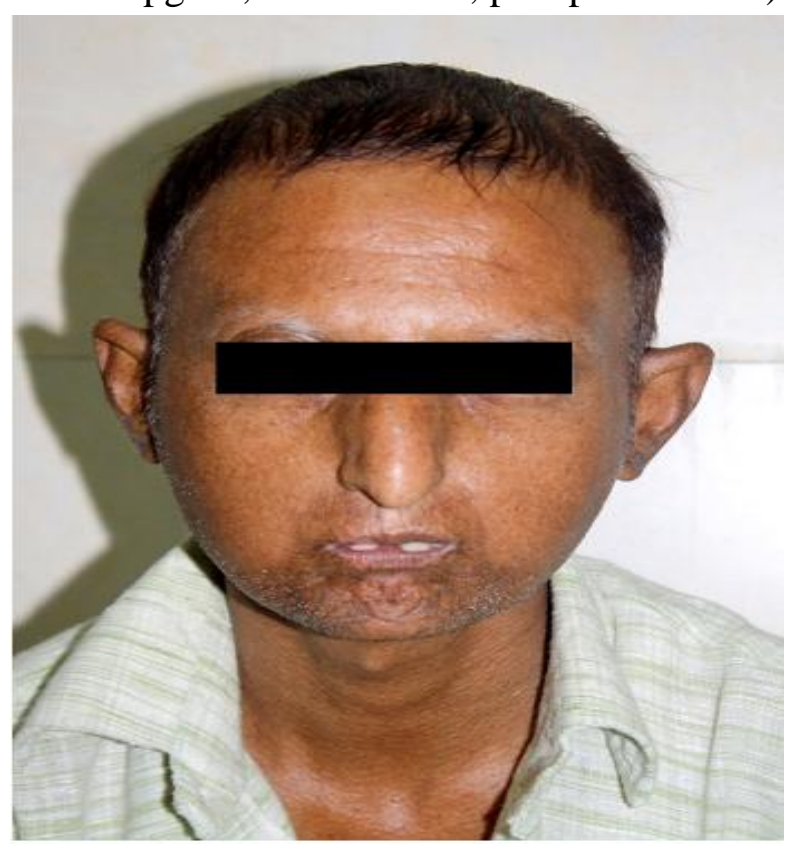

Figure 1- Dubowitz Syndrome patient frontal view

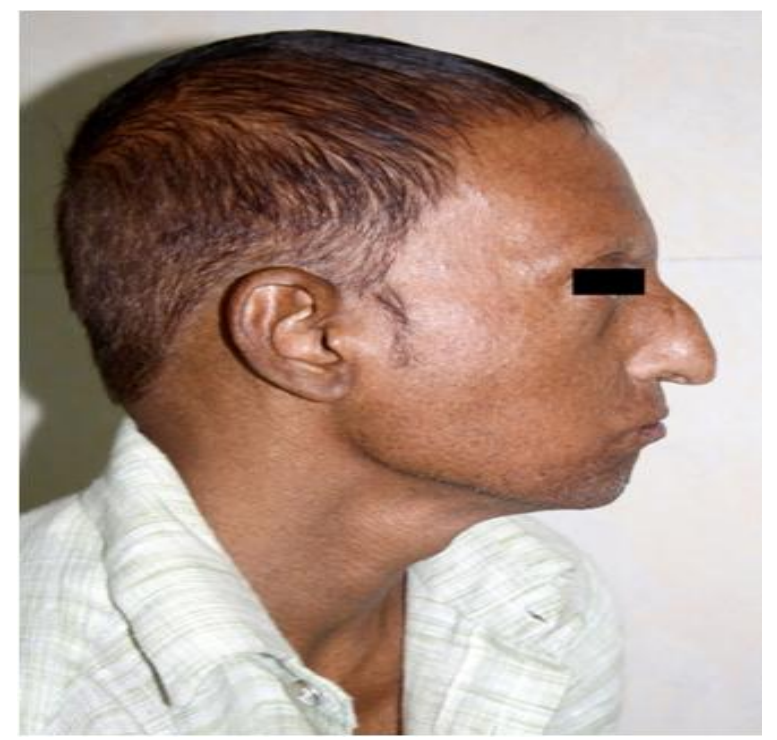

Figure 2- Dubowitz Syndrome patient lateral view

\section{Investigation}

\begin{tabular}{|c|c|c|c|c|}
\hline S.no & Test name & Result & Units & Bio.ref.interval \\
\hline 1. & Haemoglobin & $8.4 \downarrow$ & $\mathrm{g} / \mathrm{dl}$ & $14-18$ \\
\hline 2. & $\mathrm{Mcv}$ & $58 \downarrow$ & $\mathrm{fl}$ & $75-95$ \\
\hline 3. & Mch & $18 \downarrow$ & $\mathrm{pg}$ & $27-32$ \\
\hline 4. & Mchc & $29 \downarrow$ & $\%$ & $30-32$ \\
\hline 5. & Iron & $20 \downarrow$ & $\mathrm{ug} / \mathrm{dl}$ & $50-170$ \\
\hline 6. & Tibc & $543 \uparrow$ & $\mathrm{ug} / \mathrm{dl}$ & $250-425$ \\
\hline 7. & $\begin{array}{c}\text { Transferrin } \\
\text { saturation }\end{array}$ & $3.68 \downarrow$ & $\%$ & $15-50$ \\
\hline 8. & Ferritn & $4.60 \downarrow$ & $\mathrm{ng} / \mathrm{ml}$ & $10-291$ \\
\hline 9. & Hba1c & $9.5 \uparrow$ & $\%$ & $<6.5$ \\
\hline 10. & Tsh & $75.04 \uparrow$ & $\mathrm{uIU} / \mathrm{ml}$ & $0.35-5.60$ \\
\hline 11. & $\mathrm{~T} 3$ total & $.58 \downarrow$ & $\mathrm{Ng} / \mathrm{ml}$ & $.60-1.81$ \\
\hline 12 & T4 total & $2.10 \downarrow$ & $\mathrm{ug} / \mathrm{ml}$ & $5.01-12.45$ \\
\hline 13. & Anti tpo antibody & $100 \uparrow$ & $\mathrm{IU} / \mathrm{ml}$ & $<35 \mathrm{IU} / \mathrm{ml}$ \\
\hline 14. & $\begin{array}{c}\text { Intact parathyroid } \\
\text { hormone }\end{array}$ & $\begin{array}{c}12.80 \\
\downarrow\end{array}$ & $\mathrm{pg} / \mathrm{ml}$ & $14-72$ \\
\hline 15. & Calcium & $7.6 \downarrow$ & $\mathrm{mg} / \mathrm{dl}$ & $8.8-10.60$ \\
\hline 16. & Phosphorus & $6.64 \uparrow$ & $\mathrm{Mg} / \mathrm{dl}$ & $2.40-4.40$ \\
\hline 17. & $\begin{array}{c}\text { Provocative } \\
\text { growth hormone }\end{array}$ & $0.45 \downarrow$ & $\mathrm{Ng} / \mathrm{ml}$ & $>5$ \\
\hline 18. & Serum cortisol & $\begin{array}{c}15.16 \\
\mathrm{~N} \\
\end{array}$ & $\mathrm{ug} / \mathrm{dl}$ & $4.30-22.40$ \\
\hline 19. & $\begin{array}{c}\text { Serum insulin } \\
\text { fasting }\end{array}$ & $\begin{array}{c}29.40 \\
\uparrow \\
\end{array}$ & $\mathrm{uU} / \mathrm{ml}$ & $2.00-25.00$ \\
\hline 20. & $\begin{array}{c}\text { Serum ttg } \\
\text { antibody }\end{array}$ & $6.64 \mathrm{~N}$ & units & $<20$ \\
\hline 21. & Serum iga & $237 \mathrm{~N}$ & $\mathrm{Mg} / \mathrm{dl}$ & $70-400$ \\
\hline 22. & $\begin{array}{c}\text { Serum } \\
\text { testosterone total }\end{array}$ & $\begin{array}{c}270.79 \\
\mathrm{~N} \\
\end{array}$ & $\mathrm{ng} / \mathrm{dl}$ & $175-781$ \\
\hline
\end{tabular}

Echocardiography- normal left ventricle systolic function (lvef-60\%), x-ray skull suggestive of micrognathia of mandible.

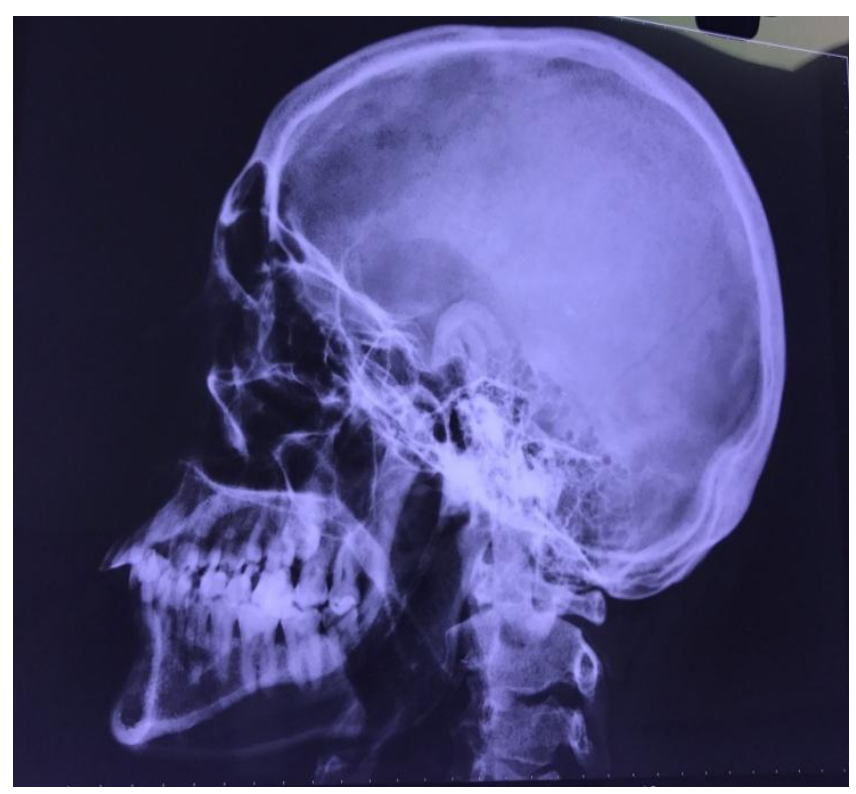

Figure 3- x-ray skull lateral view 

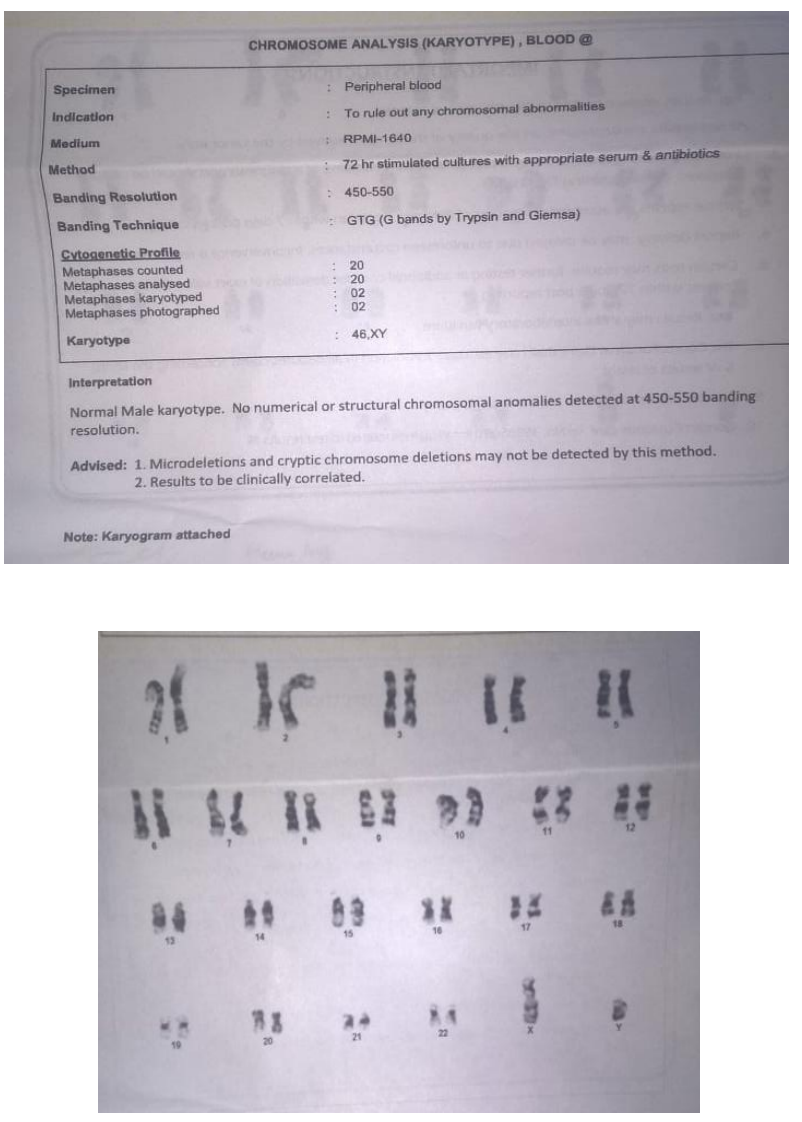

Figure-4 chromosomal analysis (karyotype), blood

\section{Discussion}

The constellation of features in this patient was consistent with the diagnosis of dubowitz syndrome. This syndrome was first described in 1965 by English physian Victor Dubowitz. ${ }^{4}$ There is no precise medical test that will conclusively consign the diagnosis of this syndrome since genetic reason is unknown. ${ }^{5}$ Apart from the characteristic facies, a variety of ocular, dental, cutaneous, skeletal, cardiovascular, gastrointestinal, neurological, immunological and hematological difficulties have been described. Other features are- partial webbing of finger, eczema $50 \%$, sacro coccygeal anomaly undescended / ectopic testes, persistent low cholesterol level. ${ }^{6}$

Polyendocrinal involvement occur likes diabetes mellitus, autoimmune hypothyroidism, hypoparathyroidism. Patient also found iron deficiency anemia with growth hormone deficiency.

\section{Conclusion}

Prompt diagnosis with regular follow up and supportive care remains the key to the management of this disorder. For involvement of endocrine gland treatment is replacement of hormone and according to investigation.

\section{References}

1- "Dubowitz syndrome | Genetic and Rare Diseases Information Center (GARD) - an NCATS Program". rarediseases.info.nih.gov. Retrieved 201707-11.

2- S. Takahira, T. Kondoh, M. Sumi et al., "Klippel-Feil anomaly in a boy and Dubowitz syndrome with vertebral fusion in his brother: a new variant of Dubowitz syndrome?" American Journal of Medical Genetics, vol. 138, no. 3, pp. 297-299, 2005.

3- Tsukahara M, Opitz JM. Dubowitz syndrome: review of 141 cases including 36 previously unreported patients. Am J Med Genet. 1996;63,1:277-289.

4- Dubowitz V. Familial Low Birthweight Dwarfism with an Unusual Facies and a Skin Eruption. J Med Genet.1965;42: 12-17.

5- Pascual JC, Betlloch I, Banuls J, et al. What syndrome is this? Dubowitz syndrome. Pediatr Dermatol. 2005;22,5:480-481.

6- Online Mendelian Inheritance in Man (OMIM) Rasmussen, Sonja A. Dubowitz Syndrome $-223370$ (https://omim.org/entry/223370). 